\title{
A primeira experiência profissional do enfermeiro na Atenção Básica
}

\section{The first professional experience of nurses in Primary Care}

\author{
Ernandes Gonçalves Dias' ${ }^{1}$ (1) \\ Carolina Cardoso dos Anjos ${ }^{2}$ (i) \\ Leandro Pereira da Silva ${ }^{3}$ (1) \\ Lyliane Martins Campos ${ }^{4}$ \\ Maiza Barbosa Caldeira ${ }^{5}$ (1)
}

${ }^{1}$ Autor para correspondência. Faculdade Verde Norte (Mato Verde). Minas Gerais, Brasil. ernandesgdias@yahoo.com.br 2-5Faculdade Verde Norte (Mato Verde). Minas Gerais, Brasil. carolanjjos@yahoo.com, leopedasilva@hotmail.com, lyliport@gmail.com, maizacaldeira@yahoo.com.br

RESUMO | OBJETIVO: descrever a primeira experiência profissional do enfermeiro na Atenção Básica. MÉTODo: estudo descritivo de abordagem qualitativa. Os dados foram coletados no período de agosto a setembro de 2019 por meio de uma entrevista semisestruturada aplicada a nove enfermeiros atuantes em Unidades de Saúde da Família de Monte Azul, Minas Gerais. RESULTADOS: a primeira experiência profissional na Atenção Básica é um desafio e oportunidade para afirmação da identidade profissional. Gera ansiedade, medo e insegurança. As dificuldades têm relação com a pouca familiaridade dos profissionais com os atributos da Atenção Básica, ao gerenciamento e ao cuidado assistencial. CONCLUSÃO: apesar de ser um desafio a primeira experiência profissional na Atenção Básica pode oportunizar ao Enfermeiro o desenvolvimento e aprimoramento de competências e habilidades fundamentais para o crescimento profissional, mesmo quando o profissional já tem outra experiência profissional que não neste campo de trabalho.

DESCRITORES: Enfermeiras e enfermeiros. Exercício profissional. Atenção Primária à Saúde. Mercado de trabalho. Desempenho profissional.

\begin{abstract}
OBJECTIVE: describe the nurse's first professional experience in Primary Care. METHOD: this is a descriptive study with a qualitative approach. Data were collected from August to September 2019 through a semi-structured interview applied to nine nurses working in Family Health Units in Monte Azul, Minas Gerais, Brazil. RESULTS: the first professional experience in Primary Care is a challenge and an opportunity to affirm professional identity. It generates anxiety, fear and insecurity. The difficulties are related to the professionals' lack of familiarity with the attributes of Primary Care, management and care. CONCLUSION: despite being a challenge, the first professional experience in Primary Care can provide the Nurse with the opportunity to develop and improve skills and abilities that are essential for professional growth, even when the professional already has a professional experience other than in this field of work.
\end{abstract}

DESCRIPTORS: Nurses. Primary Health Care. Professional practice. Job market. Work performance. 


\section{Introdução}

Depois de formados os profissionais têm como desafio a conquista do primeiro emprego, a primeira experiência profissional ${ }^{1}$. As contratações de enfermeiros, ainda inexperientes, acontecem em maior parte para atuar em serviços de saúde como as Unidades de Saúde da Família (USF) que compõem a Atenção Básica (AB), onde o profissional se insere na Estratégia Saúde da Família (ESF) nos diversos municípios do Brasil2.

A AB é caracterizada pelo conjunto de ações de saúde em nível individual e coletivo para promover e proteger a saúde, prevenir, diagnosticar e tratar agravos, cuidados paliativos, vigilância em saúde, reabilitar e reduzir danos, que se desenvolve a partir de práticas de cuidado integradas, gestão qualificada, processada por equipe constituída de multiprofissionais à população de um determinado território ${ }^{3}$.

Em expansão no Brasil, a AB tem como desafio ser implementada na perspectiva de ser um modelo assistencial focado nas necessidades de saúde apresentadas pela população ${ }^{4}$. Tem como atributos essenciais o primeiro contato, a longitudinalidade, a integralidade e a coordenação do cuidado, papeis amplamente gerenciados por enfermeiros ${ }^{5-6}$.

Nesse sentido, o enfermeiro é peça-chave na promoção e manutenção da saúde, na prevenção de doenças e na educação em saúde ${ }^{4}$, por isso é importante a atuação desse profissional nas equipes de $A B$ em todo território nacional. Entre as atribuições do enfermeiro que atua na $A B$ estão as atividades de assistência, gestão, territorialização e cuidado continuado à população adscrita, acolhimento, busca ativa, notificação e investigação de casos, reuniões, gerenciamento de insumos, materiais e pessoas, educação em saúde, dentre outras ${ }^{?}$.

Em um cenário de múltiplas atribuições o enfermeiro iniciante é constantemente desafiado a desenvolver estratégias de enfrentamento para gerenciar o estresse decorrente da divergência entre a aprendizagem da vida acadêmica e a prática profissional. Os desafios ainda perpassam pelo desenvolvimento do papel de líder da equipe, gestão e habilidades técnicopolíticas, além do aprimoramento de competências e habilidades técnicas no desempenho de papéis na esfera da saúde ${ }^{8}$.
A motivação para realizar este estudo surgiu a partir dos relatos cotidianos de sentimentos e expectativas geradas nos graduandos em Enfermagem em relação aos desafios profissionais após a formatura, assim, como a forma de preparar os novos profissionais para a primeira experiência profissional na $A B$ é fundamental conhecer a realidade vivenciada por Enfermeiros quando iniciaram sua atividade profissional nesse ambiente.

Ampliar o conhecimento em relação à primeira experiência profissional na $A B$ poderá produzir evidências que poderão nortear o ensino da enfermagem e políticas de educação permanente no trabalho e, servir de reflexão para gestores, instituições de ensino, estudantes e profissionais de Enfermagem quanto à atuação profissional.

Destarte, este estudo teve como questão norteadora: como é a primeira experiência profissional do enfermeiro na $A B$ ? Desse modo, o estudo teve como objetivo descrever a primeira experiência profissional do enfermeiro na $A B$.

\section{Método}

Trata-se de um estudo descritivo de abordagem qualitativa realizado com nove enfermeiros atuantes em sete USF de Monte Azul, Minas Gerais.

Considerou-se como critério de inclusão para participar do estudo ser Enfermeiro atuante nas ESF há no mínimo seis meses até o momento da coleta de dados, independentemente de o emprego atual na $A B$ ser a primeira experiência do profissional. Este tempo mínimo de experiência na $A B$ é considerado fundamental para que o profissional já tenha vivido situações suficientes para contribuir com a pesquisa. Foram exclusos aqueles que estavam afastados do serviço, por qualquer motivo, durante a coleta de dados ou que não foi possível coletar os dados em até três tentativas. Do universo de 11 enfermeiros, nove foram elegíveis.

Foi utilizado como instrumento de coleta de dados um roteiro semiestruturado com questões disparadoras: quais foram as dificuldades iniciais para atuação profissional em sua primeira experiência na $A B$ ? 
Quais sentimentos foram manifestados durante a fase inicial de atuação profissional na $A B$ ?

Os dados foram coletados no período de agosto a setembro de 2019, a partir de uma entrevista agendada previamente, aplicada em sala privativa na USF. As entrevistas foram gravadas em áudio por meio de aplicativo de voz, posteriormente, transcritas na íntegra, categorizadas e analisadas de acordo a técnica Análise de Temática na perspectiva de Braun e Clarke ${ }^{9}$, a partir de duas categorias para análise.

O tempo médio de duração das entrevistas foi de 15 minutos. A identidade dos entrevistados foi preservada com a substituição de seus nomes pelo nome de flores na apresentação do conteúdo.

Todos os procedimentos metodológicos obedeceram aos padrões estabelecidos pela Resolução 466/2012, do Conselho Nacional de Saúde, o projeto de pesquisa do estudo foi aprovado pelo Comitê de Ética em Pesquisa da Universidade Estadual de Montes Claros pelo Parecer Consubstanciado $\mathrm{n}$. 16409419.4.0000.5146. Todos os participantes assinaram um Termo de Consentimento Livre e Esclarecido para consentir o uso dos dados coletados.

\section{Resultados e Discussão}

O estudo foi realizado com nove enfermeiros, oito do sexo feminino, com variação de idade entre 28 e 41 anos. $O$ tempo médio de atuação na $A B$ era de seis anos. Para seis participantes o emprego atual na $A B$, foi a primeira experiência profissional e três já tiveram outra experiência profissional na enfermagem antes de atuar na $A B$.

Três profissionais tinham mais de um vínculo empregatício na área de enfermagem. É importante ressaltar que o ingresso dos participantes, no atual trabaIho na $A B$ foi mediante concurso público realizado no ano de 2015.

A análise do material empírico possibilitou a identificação de duas categorias de análise, "Sentimentos gerados na primeira experiência profissional na $A B$ " e "Dificuldades enfrentadas para o exercício profissional na primeira experiência na $A B^{\prime \prime}$.

\section{Sentimentos gerados na primeira experiência profissional na AB}

Conforme relatos dos participantes, especialmente entre aqueles que já tinham outras experiências profissionais que não na $A B$, a primeira experiência profissional neste campo de trabalho constituiu-se em um momento desafiador, sobretudo de afirmação da identidade profissional sob a luz da deontologia da enfermagem:

[...] ter que fazer ações, ou ser induzida, solicitada de executar procedimentos que não era da competência da enfermagem né, isso me neguei fazer, que era suturar o paciente [...]. Rosa

Os artigos 22 e 62 da Resolução 564 de 2017 do Conselho Federal de Enfermagem garantem, respectivamente, ao profissional de enfermagem o direito de recusar-se e proíbe de executar, atividades que não sejam de sua competência técnico-cientíca, ética e legal10.

Desafios dessa natureza, enfrentados pelos profissionais podem ser considerados como um incitamento à superação de circunstâncias adversas, assim, possibilita mudança de tal situação e contribui para o cres-

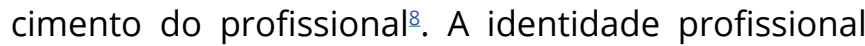
do enfermeiro é conquistada na medida em que há maior visibilidade e sentimento de pertencimento a uma categoria específica, isso viabiliza a definição de competências, autonomia e reconhecimento profissional e respaldo para tomar decisões ${ }^{11}$.

Entre os enfermeiros, em situação de primeiro emprego, houve relatos de ansiedade, medo e expectativas geradas pelo fato de realizar tarefas não mais sob supervisão dos professores. A figura do professor supervisor em atividades práticas constitui um porto seguro enquanto graduandos, ao passo que sua ausência torna a primeira experiência profissional na $A B$ estressante.

No primeiro ano foi bem difícil, primeiro porque não tinha mais o professor ou qualquer outra pessoa para me socorrer nos momentos de dúvidas, isso gera medo [...]. Tulipa

[...] eu tinha muita expectativa, então é claro que a gente fica um pouco ansiosa, por estar ali sozinha sem os professores [...]. Margarida 
O papel do enfermeiro professor é facilitar o movimento do processo de ensinar e aprender, e nesse movimento levar o estudante para mais próximo da prática profissional, para desenvolver as habilidades necessárias 12 .

A mudança da faculdade para a atuação profissional gera momentos de vulnerabilidade, pois não haverá um docente responsável, tendo assim que enfrentar as situações sozinho ${ }^{13}$. Por ser um processo desafiador, muitas vezes, por ter que assumir novas responsabilidades, novas rotinas e competências surgem sentimentos de ansiedade, medo e insegurança ${ }^{1}$.

Destaca-se também que na primeira experiência profissional, existe insegurança relacionada à inexperiência, receio em desenvolver cuidados e procedimentos técnicos, em administrar uma Unidade de Saúde, pois encontram dificuldades em liderar a equipe ${ }^{10}$.

\section{Dificuldades enfrentadas para o exercício profis- sional na primeira experiência na $A B$}

A falta de experiência na atuação em um serviço com diretrizes tão singulares como previstas para a AB demarcou as principais dificuldades encontradas pelos enfermeiros para atuação em sua primeira experiência profissional neste campo de trabalho, mesmo entre aqueles que já tinham experiência assistencial em outras áreas de atuação, como hospitais, por exemplo:

[...] eu era acostumada a trabalhar com urgência e emergência, na Atenção Básica a gente trabalha muito com a parte burocrática, faz um atendimento continuo à população, diferente da urgência e emergência, onde eu era acostumada a prestar atendimento de primeiros socorros [...] tive um pouco de dificuldade nesse início por conta dessa experiência que não tinha. Azaléia

Apesar de ainda ser um gargalo, a atenção integral, o vínculo e a coordenação do cuidado são atributos do modelo de $A B$ implantada no Brasil, que é reconhecida como ponto da rede de serviços de saúde capaz de responder às necessidades de saúde da população ${ }^{14}$.

A continuidade do cuidado, cuidado longitudinal, parece estranho ao profissional com experiência assistencial em urgência e emergência, ao passo que o profissional ingressante na $A B$ sem outras experiências assistenciais referiram a dificuldades em realizar procedimentos técnicos:
[...] não tinha experiência nenhuma quando comecei a trabalhar, este é meu primeiro emprego, quando passei no concurso havia mais de cinco anos que eu havia me graduado, então tive muitas dificuldades em alguns procedimentos técnicos [....]. Girassol

Grande parte dos enfermeiros inexperientes no ambiente de trabalho têm dificuldades durante sua atuação profissional, dentre elas cita-se as dificuldades relacionadas à falta de prática e confiança em realizar o cuidado e insegurança em realizar procedimentos técnicos ${ }^{10}$.

Percebeu-se relatos de dificuldades em gerenciar e também associados à prestação de cuidados aos usuários do serviço. As dificuldades referidas parecem ser perenes dentro do serviço, perpassaram desde falta de insumos básicos ao desafio de prestar cuidado com equipe mínima:

As dificuldades de gerenciamento eram e ainda permanecem relacionadas aos recursos humanos $e$ a necessidade de mediar conflitos, já para prestar assistência, as dificuldades são relacionadas a recursos para atender a população conforme a necessidade, a falta de equipamento de proteção individual e também,

é, por muitas vezes, de ter a equipe incompleta, com a falta de algum profissional, como a do médico. Camélia

Apesar de referida pelos profissionais como uma dificuldade percebida em sua primeira experiência na $A B$, este tipo de dificuldade parece ser independente de ser ou não a primeira experiência do profissional como apontado em estudos nessa área realizados no Brasil15-16.

Esta percepção tem relação com o fato de o enfermeiro, enquanto membro de uma equipe de saúde, desempenhar papel de liderança no grupo, liderança que se estende às ações gerenciais e assistenciais. $\mathrm{Na}$ realização de suas tarefas o profissional torna-se encarregado pelo gerenciamento técnico da equipe de enfermagem, concilia conflitos, tal como é referência aos demais membros da Equipe ${ }^{17}$.

Frisa-se, então, que ainda há muitos desafios a serem enfrentados para o trabalho na $A B$ que perpassam o trabalho em equipe, a adequação dos recursos humanos à quantidade de população atendida e valorização do profissional ${ }^{18}$. 
Há profissionais que relacionaram as dificuldades encontradas ao aprendizado durante a graduação, tiveram a percepção de haver uma dissociação entre a teoria estudada em sala e a prática assistencial:

\section{[...] como eu não tinha tanta experiência tive} dificuldade porque na faculdade você sabe como que é, é bem diferente, quando a gente vai começar a trabalhar é totalmente diferente [...]. Violeta

A deficiência que se observa na formação do profissional enfermeiro relaciona-se na capacidade em associar a teoria à prática. Durante a formação falta contato do estudante com a realidade clínica, isso pode gerar um déficit de conhecimento em certas áreas de atuação o que provoca desconforto e constrangimento ao profissional ${ }^{19}$.

Em um estudo realizado em 27 escolas de enfermagem identificou que o ensino das competências para a promoção da saúde durante a graduação do enfermeiro é regida pelo modelo hegemônico situado em componentes curriculares e na dissociação entre teoria e prática ${ }^{20}$.

Nesse sentido, reconhecem a importância da constante atualização dos conhecimentos, visto que na área da saúde ocorrem mudanças frequentes:

\section{[...] acredito que o profissional sempre teve lacunas, então sempre tem que tá fazendo educação continuada, fazendo cursos, estudando, lendo pra suprir essas lacunas [...]. Margarida}

Nesse cenário de exigências o enfermeiro é cobrado a prestar um serviço com qualidade e eficiência, desta maneira é importante que o profissional realize educação continuada, a fim buscar conhecimentos com uma visão generalista, analítica, reflexiva e humanista traçada no rigor científico, ético e intelectual21.

A educação continuada é indispensável para prestar assistência de qualidade, pois se trata de um meio de atualização técnico-científica. Paralelamente, a educação permanente se caracteriza como aprendizagem no próprio trabalho, onde o aprender e o ensinar são agregados ao ambiente profissional. Assim, tem-se a educação permanente com os desafios da prática, e a educação continuada com o desenvolvimento profissional através dos conhecimentos teóricos adquiridos 22 .
O estudo tem como limitações a quantidade de profissionais participantes e a própria natureza da pesquisa que não possibilita a generalização dos resultados, no entanto, representa um avanço no conhecimento no sentido de que traz elementos importantes para teorização pertinente ao problema investigado.

\section{Considerações finais}

A primeira experiência profissional na $A B$ é um momento desafiador na vida do profissional, mas é uma oportunidade para a construção da identidade profissional, gera sentimentos como medo, ansiedade e insegurança. Os atributos do cuidado peculiares a $A B$ como a longitudinalidade do cuidado e o vínculo tornam esta instância de atenção única, as experiências prévias em outras áreas assistenciais contribuem pouco quando o profissional se insere pela primeira vez na $A B$.

A inexperiência acarreta nos profissionais receios atrelados à consolidação do que foi aprendido em sala e com a realização dos estágios pela dificuldade de implementar a associação da teoria e prática no cuidado assistencial e gerenciamento. Contudo, não foi objetivo desse estudo demonstrar como a falta de experiência influencia na atuação do profissional, todavia poderá servir como base para outros estudos.

Apesar de ser um desafio a primeira experiência profissional na $A B$ pode oportunizar ao Enfermeiro o desenvolvimento e aprimoramento de competências e habilidades fundamentais para o crescimento profissional, mesmo quando o profissional já tenha outra experiência profissional que não neste campo de trabalho.

Desta forma, espera-se que este estudo possibilite aos profissionais, acadêmicos e gestores das instituições de ensino reflexão acerca da formação do profissional de enfermagem, para em conjunto construírem e institucionalizarem planos de ação que possa minimizar as dificuldades, tendo em vista a relevância da segurança e autoestima do profissional para realizar suas tarefas, sobretudo para que a assistência desse profissional tenha qualidade e seja pautada na evidência, ética e responsabilidade com a saúde dos usuários. 


\section{Contribuições dos autores}

Dias EG participou da concepção, delineamento, análise dos dados, interpretação dos resultados e redação do artigo científico. Anjos CC e Silva LP participaram da concepção e delineamento da pesquisa, coleta de dados, interpretação dos resultados, redação do artigo científico. Campos LM e Caldeira MB participaram do delineamento, discussão e interpretação dos resultados e revisão do artigo.

\section{Conflitos de interesses}

Nenhum conflito financeiro, legal ou político envolvendo terceiros (governo, empresas e fundações privadas, etc.) foi declarado para nenhum aspecto do trabalho submetido (incluindo, mas não se limitando a subvenções e financiamentos, participação em conselho consultivo, desenho de estudo, preparação de manuscrito, análise estatística, etc.).

\section{Referências}

1. Jesus BH, Gomes DC, Spillere LBB, Prado ML, Canever BP. Inserção no mercado de trabalho: trajetória de egressos de um curso de graduação em enfermagem. Esc Anna Nery. 2013;17(2):336-345. doi: 10.1590/S1414-81452013000200019

2. Conselho Federal de Enfermagem. Mercado de trabalho para Enfermagem amplia áreas de atuação [Internet]. 2018. Disponível em: http://www.cofen.gov.br/mercado-de-trabalho-paraenfermagem-amplia-areas-de-atuacao_65154.html

3. Brasil. Ministério da Saúde. Portaria 2.436 de 21 de setembro de 2017. Aprova a Política Nacional de Atenção Básica, estabelecendo a revisão de diretrizes para a organização da Atenção Básica, no âmbito do Sistema Único de Saúde (SUS). Brasília: Ministério da Saúde; 2017.

4. Barbiani R, Nora CRD, Schaefer R. Nursing practices in the primary health care context: a scoping review. Rev Latino-Am Enferm. 2016;24:e2721. doi: 10.1590/1518-8345.0880.2721

5. Lima JG, Giovanella L, Fausto MCR, Bousquat A, Silva EV. Atributos essenciais da Atenção Primária à Saúde: resultados nacionais do PMAQ-AB. Saúde Debate. 2018;42(spe1):52-66. doi: 10.1590/0103-11042018S104

6. Treviso P, Peres SC, Silva AD, Santos AA. Competências do enfermeiro na gestão do cuidado. Rev Adm Saúde. 2017;17(69). doi: $10.23973 /$ ras.69.59

7. Batista CC, Santos E, Ferro MRC, Araújo JC. Atribuições do enfermeiro na atenção primária: uma revisão literária. In: International Nursing Congress: Theme: Good practices of nursing representations in the construction of society. Universidade Tiradentes - Unit; 2017.
8. Oliveira WA. Enfermagem: os desafios e dificuldades do início de carreira. Refaci. 2017;2(2):1-19.

9. Braun V, Clarken V. Using thematic analysis in psychology. Qualitative research in psychology. 2006;3(2):77-101. doi: 10.1191/1478088706qp063oa

10. Conselho Federal de Enfermagem. Resolução COFEN Nº 564/2017. Código de Ética dos Profissionais de Enfermagem. DOU №233, 06 dez. Brasília; 2017.

11. Fernandes MC, Silva LMS, Silva MRF, Torres RAM, Dias MSA, Moreira TMM. Identity of primary health care nurses: perception of "doing everything". Rev Bras Enferm. 2018;71(1):142-7. doi: 10.1590/0034-7167-2016-0382

12. Pereira LGM, Cardoso AL. A formação profissional do enfermeiro docente, que atua no ensino técnico: e o saber formar profissionais capazes de pensar e gestar soluções. Rev UNINGÁ. 2017;54(1):79-90.

13. Mattosinho MMS, Coelho MS, Meirelles BHS, Souza SS, Argente CE. Mundo do trabalho: Alguns aspectos vivenciados pelos profissionais recém-formados em enfermagem. Acta Paul Enferm. 2010;23(4):446-41. doi: 10.1590/S0103-21002010000400004

14. Figueiredo DCMM, Shimizu HE, Ramalho WM, Figueiredo AM, Lucena KDT. Quality of Primary Health Care in Brazil: patients' view. Rev Bras Enferm. 2018;71(Suppl 6):2713-9. doi: 10.1590/0034-7167-2017-0656

15. Teixeira NL, Silva MM, Draganov PB. Desafios do enfermeiro no gerenciamento de conflitos dentro da equipe de enfermagem. Rev Adm Saúde. 2018;18(73). doi: 10.23973/ras.73.138

16. Leonelli LB, Andreoni S, Martins P, Kozasa EH, Salvo VL, Sopezki D et al. Perceived stress among Primary Health Care Professionals in Brazil. Rev Bras Epidemiol. 2017;20(2):286-298. doi: 10.1590/1980-5497201700020009

17. Rocha GSA, Andrade MS, Silva DMR, Terra MG, Medeiros SEG, Aquino JM. Feelings of pleasure of nurses working in primary care. Rev Bras Enferm. 2019;72(4):1093-100. doi: 10.1590/0034-71672018-0518

18. Braghetto GT, Sousa LA, Beretta D, Vendramini SHF. Dificuldades e facilidades do enfermeiro da Saúde da Família no processo de trabalho. Cad Saúde Colet. 2019;27(4):420-426. doi: $\underline{10.1590 / 1414-462 \times 201900040100}$

19. Melo RCCP, Queirós PJ, Tanaka LH, Costa PJ, Bogalho CID, Oliveira PISF. Dificuldades dos estudantes do curso de licenciatura de enfermagem no ensino clínico: percepção das principais causas. Rev Enf Ref. 2017;4(15):55-64. doi: 10.12707/RIV17059

20. Silva KL, Barcelos BJ, França BD, Araújo FL, Magalhães Neta $I T$, Ledo MM. Between experiments and experiences: challenges for teaching competencies for health promotion in Nursing Education. Interface. 2018;22(67):1209-20. doi: 10.1590/180757622017.0467 
21. Puschel VAA, Costa D, Reis PP, Oliveira LB, Carbogim FC.

Nurses in the labor market: professional insertion, competencies and skills. Rev Bras Enferm. 2017;70(6):1288-1295. doi:

10.1590/0034-7167-2016-0061

22. Arcanjo RVG, Chistovam BP, Braga ALS. Recomendações sobre exposição aos riscos ocupacionais pela equipe de enfermagem: uma revisão integrativa. Rev Enferm Atual. 2017;83(21):94-101. doi: 10.31011/reaid-2017-v.83-n.21-art.291 